\title{
Increasing rates of SSA/P detection in a large open-access Australian colonoscopy cohort
}

\section{(ㄷ)(1)}

Authors

Kumanan Nalankilli ${ }^{1,2}$, Xuan Thanh Huynh ${ }^{1}$, Stephen Lade ${ }^{3}$, Mark Stephens ${ }^{4}$, Robert Hewett ${ }^{4}$, Alan Moss ${ }^{1,2,4}$

Institutions

1 Department of Endoscopic Services, Western Health, Melbourne, Australia

2 Department of Medicine - Western Health, Melbourne Medical School, The University of Melbourne, Victoria, Australia

3 Department of Anatomical Pathology, St John of God Pathology, Victoria, Australia

4 Chesterville Endoscopy, Chesterville Day Hospital, Cheltenham, Victoria, Australia

submitted 2.3.2018

accepted after revision 10.10 .2018

Bibliography

DOI https://doi.org/10.1055/a-0808-3523 |

Endoscopy International Open 2019; 07: E310-E316

(c) Georg Thieme Verlag KG Stuttgart · New York

ISSN 2364-3722

Corresponding author

Alan Moss, Director of Endoscopic Services, Western Health, Gordon St, Footscray 3011, Melbourne, Victoria, Australia

Fax: +61383457378

alan.moss@wh.org.au

\section{ABSTRACT}

Background and study aims There are limited longitudinal data regarding detection rates for sessile serrated adenoma/polyps (SSADR) and right-sided hyperplastic polyps (RHPDR) that constitute the proximal serrated lesion detection rate (PSLDR). Recently, a minimum PSLDR of $4.5 \%$ has been suggested. This study was designed to assess SSADR, PSLDR and adenoma detection rate (ADR) for a newly quali- fied gastroenterologist and compare them to published data and to assess the change in SSADR, PSLDR and ADR over time for potential improvement with experience.

Patients and methods All colonoscopies performed by a single colonoscopist (AM), at one Australian ambulatory direct-access endoscopy center over 4 years from 2011 to 2015 were retrospectively analyzed. Histology was reported by a single expert pathologist (SL). ADR, SSADR, RHPDR and PSLDR were recorded.

Results A total of 841 colonoscopies were performed on 637 patients. Of them, 454 (54\%) were males. Mean age was 59 years. Of the colonoscopies, $87 \%$ were performed for patients with ASA scores of 1-2, 422 (50.2\%) were for screening or surveillance, $374(44.5 \%)$ for investigation of symptoms and 45 (5.4\%) had therapeutic indications. Conventional adenomas were detected in 346 colonoscopies $(\mathrm{ADR}=41.1 \%), \mathrm{SSA} / \mathrm{P}$ in $124(\mathrm{SSADR}=14.7 \%)$ and $\mathrm{RHP}$ in the absence of SSA/P in 35 (RHPDR $=4.2 \%$ ). PSLDR was $18.9 \%$. ADR was stable over time (range $33 \%-50 \%$ ). SSADR and PSLDR increased over time [SSADR: $8.6 \%$ (2011), 8.4\% (2012), 14.9\% (2013), 18.5\% (2014), 25.0\% (2015); PSLDR: $10.5 \%$ (2011), $11.3 \%$ (2012), $16.8 \%$ (2013), 27.2\% (2014), $29.4 \%$ (2015)]. There was a statistically significant improvement in SSADR (IRR 1.37) and PSLDR (IRR 1.36) over the study period $(P<0.001)$, whereas the ADR remained stable (IRR 1.04, $P=0.334$ ).

Conclusions SSADR and PSLDR in this unselected directaccess cohort are high and exceed previously reported detection rates in the final 2 years. Detection rates improved with experience, likely representing a learning effect. The minimum expected PSLDR may need to be revised upwards and further studies are required, particularly in areas where screening colonoscopies are offered only for patients with increased colorectal cancer risk (family history or fecal immunochemical test-positive).

\section{Introduction}

Colorectal cancer (CRC) screening and surveillance programs aim to detect and remove adenomas, on the basis that the traditional adenoma-carcinoma sequence results in the develop- ment of CRC from pre-existing adenomas [1-2]. However, it is now well recognized that other molecular pathways exist for development of CRC. The serrated neoplasia pathway accounts for up to one-third of CRC, where the sessile serrated adenoma/ polyp (SSA/P) is the principal serrated precursor lesion [3]. 
Therefore, it is important to identify and remove SSA/P when they are encountered during colonoscopy. Nevertheless, their detection and removal remain challenging.

Recently, a minimum proximal serrated lesion detection rate (PSLDR) of $4.5 \%$ has been recommended for serrated lesions inclusive of SSA/P and hyperplastic polyps proximal to the splenic flexure, for United States-based colonoscopy screening programs that involve colonoscopy for the average-risk population [4]. However, in other countries such as Australia, where screening by colonoscopy is only recommended on higher-risk populations (family history or positive fecal immunochemical test [FIT]) and not on the average-risk population [5], the PSLDR should in theory be higher. There is little data to enable minimum parameters for PSLDR to be set in general, but particularly in this setting. Therefore, evaluating the SSA/P detection rate (SSADR) and PSLDR and comparing these with conventional adenoma detection rates (ADR) is an important step, and may assist in establishing an acceptable minimum SSADR for colonoscopists. PSLDR and SSADR may also evolve into future quality markers for colonoscopy. There is limited longitudinal data assessing the effect of learning and experience on an individual's ability to detect these subtle lesions.

The current study had two aims: 1 . To assess the PSLDR, SSADR and ADR of a newly qualified gastroenterologist and compare them to published detection rates; and 2. To assess the change in PSLDR, SSADR and ADR over time to determine if there is improvement with learning and experience.

\section{Patients and methods}

All colonoscopies at an Australian ambulatory day endoscopy center (Chesterville Day Hospital) between August 2011 and August 2015 by a single endoscopist (AM) since graduating from gastroenterology training and commencing consultant practice were retrospectively audited. The audit was conducted according to the guidelines of the National Health and Medical Research Council (NHMRC) of Australia for clinical audit. All patients were referred by General Medical Practitioners directly for colonoscopy. The suburb of Cheltenham where the endoscopy center is located consists of a predominantly Caucasian but multicultural, middle socioeconomic class population. All colonoscopies were performed in the morning. Bowel preparation was with Prep-Kit C, which consists of one Glycoprep-C sachet and two PicoPrep sachets. This was administered the night prior to colonoscopy. From January 2015, this was changed to a split-dose administration, with the second of the PicoPrep sachets being administered early in the morning on the day of colonoscopy. Quality of bowel preparation for each colonoscopy was graded as good, average, or poor. Olympus 180 series high-definition (HD) colonoscopes were used from the beginning of the study until October 2012. Olympus 190 series colonoscopes were used from November 2012 onwards. All colonoscopies were performed under deep sedation using intravenous propofol, administered by a general practitioner anesthetist $(\mathrm{RH})$. Cecal retroflexion was not performed routinely. Rather, a two-pass technique past the hepatic flexure was utilized. Virtual chromoendoscopy using narrow-band imaging was not used routinely, but only to interrogate a specific area of concern. Furthermore, patient position changes were also not routinely carried out. The data that were retrospectively analyzed for this study were prospectively recorded, and included indications for colonoscopy, patient demographics, previous colonoscopy history, American Society of Anesthesiologists (ASA) score, location of polyp and histopathology result. All histology was reported prospectively by a single expert gastrointestinal pathologist (SL). Surveillance intervals were determined based on the Australian National Health and Medical Research Council Clinical Practice Guidelines for Surveillance Colonoscopy [6]. In cases where bowel preparation was not satisfactory for the indication, colonoscopy was repeated with enhanced bowel preparation, either immediately, or at an earlier surveillance interval as clinically appropriate.

\section{Statistical analysis}

Yearly detection rate for each polyp type was calculated. Factors associated with ADR, SSADR and PSLDR were evaluated using the chi-squared test. Changes in detection rate over time were analyzed for significance using Poisson regression and expressed as the incidence rate ratio (IRR). Changes over time in other baseline variables were evaluated using the chisquared test, Fisher's exact test and Rank-Sum test, and those with significant results were used for subgroup analysis. Statistical analyses were performed using IBM SSDN and Stata 14.1 software.

\section{Results \\ Demographics}

A total of 841 colonoscopies were carried out on 637 patients over the study period. Of these, 454 colonoscopies were on males (54\%) and 387 (46\%) on females. The mean age \pm SD of patients was $58.9 \pm 14.2$ years. Of the colonoscopies, 731 (87\%) were carried out for patients with an ASA score of 1 or 2 . Of the 637 patients, 487 (76.4\%) had one colonoscopy, $119(18.7 \%)$ had two colonoscopies and 31 (4.9\%) had three or more colonoscopies during the study period. Of the 841 colonoscopies, 279 (33.2\%) were for patients who previously underwent one or more colonoscopies by another colonoscopist prior to the present study period.

\section{Colonoscopes used}

Olympus 180 HD colonoscopes were used for all 292 colonoscopies (34.7\%) between August 2011 and October 2012. The subsequent 549 colonoscopies (65.3\%) from November 2012 to August 2015 were carried out using Olympus 190 series colonoscopes.

\section{Indications}

Of the 841 colonoscopies, 102 (12.1\%) were for screening (asymptomatic patients with a positive family history of bowel cancer or a positive fecal immunochemical test), 320 (38.0\%) were for surveillance for a personal history of colonic polyps and 374 (44.5\%) were for investigation of symptoms, which included presentations such as lower gastrointestinal bleeding, 
- Table 1 Lesion detection rates over the study period.

\begin{tabular}{|c|c|c|c|c|c|c|c|c|c|}
\hline Year & $\begin{array}{l}\text { Colonos- } \\
\text { copies }\end{array}$ & $\begin{array}{l}\text { Convention- } \\
\text { al adenoma } \\
\text { present in }\end{array}$ & $\begin{array}{l}\text { ADR } \\
(\%)\end{array}$ & $\begin{array}{l}\text { SSA/P } \\
\text { present } \\
\text { in }\end{array}$ & $\begin{array}{l}\text { SSADR } \\
\text { (\%) }\end{array}$ & $\begin{array}{l}\text { RHP excluding } \\
\text { synchronous } \\
\text { SSA/P present in }\end{array}$ & $\begin{array}{l}\text { RHPDR } \\
\text { (\%) }\end{array}$ & $\begin{array}{l}\text { PSL (HP+ } \\
\text { SSA/P) } \\
\text { present in }\end{array}$ & $\begin{array}{l}\text { PSLDR } \\
(\%)\end{array}$ \\
\hline 2011 & 105 & 49 & $46.7 \%$ & 9 & $8.6 \%$ & 2 & $1.9 \%$ & 11 & $10.5 \%$ \\
\hline 2012 & 238 & 90 & $37.8 \%$ & 20 & $8.4 \%$ & 7 & $2.9 \%$ & 27 & $11.3 \%$ \\
\hline 2013 & 167 & 56 & $33.5 \%$ & 25 & $15.0 \%$ & 3 & $1.8 \%$ & 28 & $16.8 \%$ \\
\hline 2014 & 195 & 83 & $42.6 \%$ & 36 & $18.5 \%$ & 17 & $8.7 \%$ & 53 & $27.2 \%$ \\
\hline 2015 & 136 & 68 & $50.0 \%$ & 34 & $25.0 \%$ & 6 & $4.4 \%$ & 40 & $29.4 \%$ \\
\hline Overall & 841 & 346 & $41.1 \%$ & 124 & $14.7 \%$ & 35 & $4.2 \%$ & 159 & $18.9 \%$ \\
\hline
\end{tabular}

ADR, adenoma detection rate; SSA/P, sessile serrated adenoma/polyp; SSADR, sessile serrated adenoma detection rate; RHP, right-sided hyperplastic polyp; RHPDR, right-sided hyperplastic polyp detection rate; PSL, proximal serrated lesion; PSLDR, proximal serrated lesion detection rate

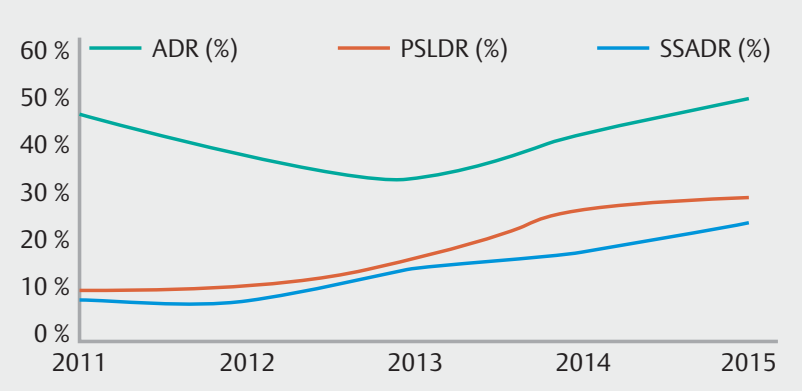

Fig. 1 Overall ADR, PSLDR and SSADR over the study period.

iron deficiency anaemia, change in bowel habit, weight loss or abdominal pain. A total of 45 colonoscopies (5.4\%) were for therapeutic reasons such as polypectomy or treatment of bleeding (e.g. due to radiation proctitis or angiodysplasia). Only one patient was known to have a familial CRC syndrome. He had HNPCC syndrome and had two colonoscopies during the study period.

\section{Lesion detection rates}

Conventional adenomas were detected in 346 of the 841 colonoscopies, corresponding to an overall ADR of $41.1 \%$. SSA/P were detected in 124 colonoscopies, corresponding to a SSADR of $14.7 \%$. Right-sided hyperplastic polyps (from the cecum, ascending and transverse colon) in the absence of synchronous SSA/P were detected in 35 colonoscopies, corresponding to a RHPDR of $4.2 \%$. The combined detection rate of SSA/P and RHP resulted in a PSLDR of $18.9 \%$. Carcinomas were detected in 8 colonoscopies ( $1 \%)$.

The ADR per year fluctuated between $33 \%$ and $50 \%$ ( $\triangleright$ Table 1 and $>$ Fig. 1). However, the SSADR showed an increasing trend over time $(8.6 \%$ in $2011,8.4 \%$ in $2012,14.9 \%$ in 2013 , $18.5 \%$ in $2014,25.0 \%$ in 2015). The PSLDR also increased each year during the study period $(10.5 \%$ in $2011,11.3 \%$ in 2012 , $16.8 \%$ in 2013, 27.2\% in 2014 and $29.4 \%$ in 2015) (ฉ Table 1 and $>$ Fig. 1). Analysis of detection rates per year showed that the ADR did not demonstrate a statistically significant change over the study period (IRR 1.04, $P=0.334$ ). However, the SSADR (IRR 1.37, $P<0.001$ ) and the PSLDR (IRR 1.36, $P<0.001)$ demonstrated a statistically significant improvement over the study period. ( $\triangleright$ Table 2$)$.

- Table 2 Change in detection rates over the study period compared using Poisson regression.

\begin{tabular}{|c|c|c|c|c|c|c|c|}
\hline & & \multicolumn{2}{|l|}{ ADR } & \multicolumn{2}{|l|}{ SSADR } & \multicolumn{2}{|l|}{ PSLDR } \\
\hline & & IRR (95\% CI) & $P$ value & IRR (95\% CI) & $P$ value & IRR (95\% CI) & $P$ value \\
\hline \multicolumn{2}{|l|}{ Overall unadjusted } & $1.04(0.96,1.13)$ & 0.334 & $1.37(1.19,1.57)$ & $<0.001$ & $1.36(1.20,1.55)$ & $<0.001$ \\
\hline \multirow[t]{3}{*}{ Indication } & Symptoms & $1.04(0.90,1.20)$ & 0.623 & $1.41(1.11,1.78)$ & 0.004 & $1.39(1.14,1.70)$ & 0.001 \\
\hline & Screening & $0.89(0.72,1.11)$ & 0.306 & $1.36(0.90,2.05)$ & 0.150 & $1.45(1.00,2.09)$ & 0.050 \\
\hline & Surveillance & $1.04(0.92,1.18)$ & 0.532 & $1.29(1.04,1.60)$ & 0.022 & $1.25(1.04,1.50)$ & 0.017 \\
\hline \multirow[t]{3}{*}{ Bowel preparation } & Good & $1.04(0.94,1.14)$ & 0.490 & $1.41(1.19,1.67)$ & $<0.001$ & $1.42(1.22,1.64)$ & $<0.001$ \\
\hline & Average & $1.09(0.92,1.29)$ & 0.339 & $1.22(0.92,1.62)$ & 0.174 & $1.16(0.91,1.48)$ & 0.224 \\
\hline & Poor & $0.98(0.73,1.30)$ & 0.867 & $1.36(0.65,2.86)$ & 0.418 & $1.55(0.84,2.86)$ & 0.161 \\
\hline
\end{tabular}

ADR, adenoma detection rate; SSADR, sessile serrated adenoma/polyp detection rate; PSLDR, proximal serrated lesion detection rate 
- Table 3 Potential confounders and change over time.

\begin{tabular}{|l|l|l|l|l|l|l|}
\hline & & $\mathbf{2 0 1 1}$ & $\mathbf{2 0 1 2}$ & $\mathbf{2 0 1 3}$ & $\mathbf{2 0 1 4}$ & $\mathbf{2 0 1 5}$ \\
\hline \multirow{2}{*}{ Indication } & Symptom & $56(53 \%)$ & $131(55 \%)$ & $74(44 \%)$ & $63(32 \%)$ & $50(37 \%)$ \\
\hline & Screening & $15(14 \%)$ & $35(15 \%)$ & $18(11 \%)$ & $22(11 \%)$ & $21(15 \%)$ \\
\hline & Surveillance & $26(25 \%)$ & $57(24 \%)$ & $67(40 \%)$ & $97(50 \%)$ & $64(47 \%)$ \\
\hline & Therapy & $8(8 \%)$ & $15(6 \%)$ & $8(5 \%)$ & $13(7 \%)$ & $1(1 \%)$ \\
\hline Gender & Male & $57(54 \%)$ & $119(50 \%)$ & $92(55 \%)$ & $111(57 \%)$ & $75(55 \%)$ \\
\hline \multirow{2}{*}{ Age (median, IQR) } & Female & $48(46 \%)$ & $119(50 \%)$ & $75(45 \%)$ & $84(43 \%)$ & $61(45 \%)$ \\
\hline Bowel preparation & Good & $71(68 \%)$ & $161(68 \%)$ & $88(53 \%)$ & $114(59 \%)$ & $109(80 \%)$ \\
\hline & Average & $18(17 \%)$ & $60(25 \%)$ & $63(38 \%)$ & $59(30 \%)$ & $23(17 \%)$ \\
\hline & Poor & $16(15 \%)$ & $17(7 \%)$ & $16(10 \%)$ & $22(11 \%)$ & $4(3 \%)$ \\
\hline
\end{tabular}

- Table 4 Distribution of age and gender.

\begin{tabular}{|l|c|c|c|}
\hline Age & Median age in years of patients with polyp (IQR) & Median age in years of patients without polyp (IQR) & $P$ value \\
\hline PSL & $60(53,67)$ & $60(50,69)$ & 0.637 \\
\hline Conventional adenomas & $63.5(57,71)$ & $55(47,65)$ & $<0.001$ \\
\hline SSA/P & $60(53,67)$ & $59(50,69)$ & 0.789 \\
\hline Gender & Males with polyps (\%) & Males without polyps (\%) & $P$ value \\
\hline PSL & $94(59.1 \%)$ & $360(52.7 \%)$ & 0.158 \\
\hline Conventional adenomas & $213(61.6 \%)$ & $241(48.6 \%)$ & $<0.001$ \\
\hline SSA P & $75(60.5 \%)$ & $379(52.8 \%)$ & 0.120 \\
\hline
\end{tabular}

PSL, proximal serrated lesion; SSA/P, sessile serrated adenoma/polyp

\section{Subgroup analysis}

Age and gender

There were no significant changes in the distribution of age and gender over the study period ( $\triangleright$ Table 3 ). Patients in whom conventional adenomas were detected were older compared to those without conventional adenomas (median age 63.5 years vs 55 years; $P<0.001$ ). However, there were no significant differences in median age between patients in whom PSL and SSA/P were detected, compared to those who did not have PSL or SSA/P ( $\bullet$ Table 4 ). There was a significantly higher proportion of males among patients with conventional adenomas compared to those without conventional adenomas $(62 \%$ vs $49 \%, P<0.001)$. However, this gender difference was not observed for PSL and SSA/P ( $\triangleright$ Table 4).

\section{Indication}

The ADR, SSADR and PSLDR were all higher in surveillance colonoscopies compared to those performed for screening or for symptoms ( $\vee$ Table 5 ). The ADR in 320 surveillance colonoscopies was $51.3 \%$ compared to $42.2 \%$ in 102 screening colonos- copies $(P<0.001)$. Of the 102 screening colonoscopies, 52 were on FIT-positive patients and 50 were on those with a positive family history of bowel cancer. The ADR was significantly higher in those who were FIT-positive compared to those who were FIT-negative ( $52 \%$ vs $32 \%, P=0.047$ ) ( $\bullet$ Table 5$)$. However, no significant differences were observed in PSLDR and SSADR between those who were FIT-positive and FIT-negative ( $\triangleright$ Table 5). The proportion of colonoscopies performed for each indication (symptoms, screening and surveillance) varied between the years of the study period ( $\bullet$ Table 3 ). However, when detection rates within each indication were analyzed over the study period, the trend of increasing PSLDR with stable ADR was again demonstrated ( $\triangleright$ Table 2 ).

\section{Cecal intubation rate and bowel preparation quality}

The cecal intubation rate was stable throughout the study, with no statistically significant difference in cecal intubation rates between the years (range $98-99 \% ; P=0.956$ ) ( $\triangleright$ Table 3 ). The quality of bowel preparation varied significantly between the years $(P=0.003)(\triangleright$ Table 3$)$. In particular, after the introduc- 
- Table 5 Lesion detection rates for each indication.

\begin{tabular}{|c|c|c|c|c|c|c|}
\hline & Symptoms (N = 374) & \multicolumn{2}{|c|}{ Screening $(N=102)$} & \multicolumn{2}{|c|}{ Surveillance $(\mathrm{N}=320)$} & $P$ value \\
\hline PSLDR & $54(14.4 \%)$ & \multicolumn{2}{|l|}{$14(13.7 \%)$} & \multicolumn{2}{|l|}{$84(26.3 \%)$} & $<0.001$ \\
\hline ADR & $110(29.4 \%)$ & \multicolumn{2}{|l|}{$48(42.2 \%)$} & \multicolumn{2}{|l|}{$164(51.3 \%)$} & $<0.001$ \\
\hline \multirow[t]{2}{*}{ SSADR } & $42(11.2 \%)$ & $10(9.8 \%)$ & & $66(20.6 \%)$ & & 0.001 \\
\hline & & FIT- screening colonoscopies $(N=50)$ & \multicolumn{2}{|c|}{ FIT + screening colonoscopies $(N=52)$} & \multicolumn{2}{|l|}{$P$ value } \\
\hline PSLDR & & $9(18.0 \%)$ & \multicolumn{2}{|l|}{$6(11.5 \%)$} & \multicolumn{2}{|l|}{0.411} \\
\hline ADR & & $16(32.0 \%)$ & \multicolumn{2}{|l|}{$27(51.9 \%)$} & \multicolumn{2}{|l|}{0.047} \\
\hline SSADR & & $7(14.0 \%)$ & \multicolumn{2}{|l|}{$4(7.7 \%)$} & \multicolumn{2}{|l|}{0.353} \\
\hline
\end{tabular}

Table 6 Lesion detection rates according to quality of bowel preparation.

\begin{tabular}{|c|c|c|c|c|c|}
\hline & Good $(N=543)$ & Average $(\mathrm{N}=223)$ & $P$ value & Poor $(N=75)$ & $P$ value \\
\hline PSLDR & $105(19.3 \%)$ & $47(21.1 \%)$ & 0.583 & $7(9.3 \%)$ & 0.073 \\
\hline ADR & $213(39.2 \%)$ & $102(45.7 \%)$ & 0.096 & $31(41.3 \%)$ & 0.250 \\
\hline SSADR & $81(14.9 \%)$ & $38(17.0 \%)$ & 0.461 & $5(6.7 \%)$ & 0.089 \\
\hline
\end{tabular}

PSLDR, proximal serrated lesion detection rate; ADR, adenoma detection rate; SSADR, sessile serrated adenoma/polyps detection rate

tion of split-dose bowel preparation at the beginning of 2015, the proportion of colonoscopies with good bowel preparation increased from $62 \%$ to $80 \%$, while those rated average reduced from $28 \%$ to $17 \%$ and those rated poor reduced from $10 \%$ to $3 \%$ $(P<0.001)$. There was a trend towards a higher PSLDR in colonoscopies where the bowel preparation was either good or average, when compared to those with poor bowel preparation, though this did not reach statistical significance (19.3\% (good) vs $21.1 \%$ (average) vs $9.3 \%$ (poor), $P=0.07$ ) ( Table 6).

\section{Discussion}

Our colonoscopist's ADR did not significantly increase over the study period ( $\mathbf{F i g}$. 1). However, the overall ADR of $41.1 \%$ is higher than the recommended ADR of $30 \%$ in male patients and $20 \%$ in female patients for average-risk screening [7]. Therefore, the stable ADR serves as a measure of internal quality control against which changes observed over time in SSADR and PSLDR can be compared. In contrast, the SSADR and PSLDR increased yearly with experience ( $\mathbf{F i g} \mathbf{1} \mathbf{1})$. The overall SSADR of $15 \%$ and PSLDR of $19 \%$ achieved in this study are high compared with many previous reports, and the most recent year's PSLDR of $29.4 \%$ is, to the best of our knowledge, the highest reported among current literature ( $\triangleright$ Table 1 ). The improvement in SSADR and PSLDR likely reflects the learning effect associated with increased exposure to serrated lesions over time, as the colonoscopist's practice expanded since graduating from the training program. This learning effect is similarly demonstrated in a Dutch study, where a significant improvement in ADR and SSADR was observed in participating colonoscopists after a standardized education program was implemented (ADR $22.5 \%$ vs $25.8 \% ; P<0.001)$ and SSADR $(10.0 \%$ vs $13.5 \%$; $P<$ $0.001)[8]$.

The credibility of our data demonstrating such high prevalence of SSA/P and PSL, is supported by a recently published Australian study, where the SSADR for a single colonoscopist over a 1-year period was $20.1 \%$ in an unselected series of consecutive outpatients [9]. However, SSA/P and PSL detection is highly endoscopist-dependent [10]. In a retrospective study from the United States evaluating 11,049 polyps found in 6681 screening and surveillance colonoscopies, the PSLDR for 15 colonoscopists ranged from $1 \%$ to $26 \%$ [10]. Compared to the highest detector, the odds of detecting PSL for individual colonoscopists ranged from 0.05 to $0.67(P<0.001)$ [10]. This study involved colonoscopy for screening of average-risk patients, so the highest detecting colonoscopist in that study may well record an even higher PSDLR in an environment where colonoscopy screening is offered only for those at increased risk of CRC. Furthermore, in a single-center Australian retrospective study that evaluated factors influencing the SSADR of multiple colonoscopists, the mean ADR was $33 \%$ but SSADR was low at only $3 \%$ (range $0-17 \%$ ) [11]. These data suggest that high ADR may not always correlate with high SSADR, and therefore detection of SSA/P may be an independent skill.

The reasons for the high SSADR and PSLDR in the current study warrant discussion. The colonoscopist in the current study has high exposure to SSA/P in his main academic hospital practice, via referral from other clinicians for endoscopic mucosal resection (EMR) of large SSA/P. This concentrated experi- 
ence may have led to better recognition of SSA/P during routine diagnostic colonoscopy, which was captured in our study.

Our inference that the increased SSADR over time was a result of learning and experience is limited by two variables that changed during the study period. First the change from 180 series colonoscopes to 190 series from November 2012 may have contributed to increased SSADR. However, this change occurred early in the study, and cannot account for the continually increasing SSADR and PSLDR (with stable ADR) that was observed during the years following this change ( $\triangleright$ Table 1 ) and suggests a learning effect independent of the colonoscope used. Second, introduction of split-dose bowel preparation in 2015 may have also contributed to increased SSADR and PSLDR in the final year of the study. However, the split-dose bowel preparation was only introduced 8 months prior to the conclusion of the study. The statistically significant trend of increasing SSADR and PSLDR with a stable ADR was present prior to changing to the split-dose preparation, which represents the majority of the study duration. Third, colonoscopy withdrawal time was not recorded. A potentially longer withdrawal time in the latter years of the study period could have confounded the increased detection rates observed. However, withdrawal time is unlikely to have changed significantly during the study period, as the importance of an adequate withdrawal time was well publicized and was appreciated by AM prior to the study commencing. Moreover, the allocated procedure times remained unchanged during the study period.

Another potential criticism of our study is that all colonoscopies, including repeat colonoscopies on the same patient, were included and not only screening procedures. However, calculating the ADR or PSLDR using only screening colonoscopies can render itself to indication bias or gaming of outcomes. Historically, ADR was only calculated based on initial colonoscopies in a screening cohort. However, more recent recommendations are that ADR be calculated for all colonoscopies in individuals aged 50 or over, except where it is performed for an emergency or where it is performed for a specific therapeutic indication [12]. A recent retrospective study assessed whether calculating ADR from screening, surveillance, and diagnostic colonoscopies (overall ADR) would alter conclusions about the performance of colonoscopists, compared to using an ADR based only on screening colonoscopies [13]. For 15 colonoscopists, screening ADRs only differed from the overall ADR by a mean of 2.6 percentage points (range $0-6.9$ percentage points). The authors concluded that utilizing the overall ADR could be a simplified measure of adenoma detection, and that it would not have a significant impact on whether a colonoscopist would meet minimal recommended threshold detection rates [13].

Our study has a number of additional limitations. The retrospective nature precluded collection of additional data for risk factors for colonic polyps such as presence of diabetes, hypertension or obesity, and therefore we could not account for these risk factors in our analysis. Furthermore, the single-operator nature of the study may limit the ability to generalize the study findings to other endoscopists or centers, and prospective or multicenter studies are required to see if these findings are replicated.
The endoscopy community is beginning to debate the merits of setting benchmarks for SSADR or PSLDR. The ADR is well established as an independent predictor of interval CRC risk after screening colonoscopy, and is therefore a strong quality marker for colonoscopy [14]. However, with emerging evidence for the role of PSL in development of CRC, we believe it is logical that a minimum PSLDR should also be considered when assessing colonoscopy quality. In a recent publication by East et. al., a minimum PSLDR of $4.5 \%$ was proposed [4]. Indeed, recently the Australian colonoscopy recertification program set a benchmark of $4 \%$ for SSADR for colonoscopists applying for reaccreditation in colonoscopy. Studies such as ours may lend further weight to potentially setting a higher benchmark. However, before SSADR or PSLDR can unequivocally be considered a strong colonoscopy quality marker, further evidence is required to prove that a higher SSADR or PSLDR correlates with a reduced rate of interval CRC.

Unlike conventional adenomas, SSA/P without cytological dysplasia are not thought to typically result in occult colonic bleeding, and therefore would not be expected to be detected by the FIT. However, SSA/P are associated with increased prevalence of synchronous conventional adenomas that are known to result in occult bleeding. In an Australian retrospective study, among FIT-positive screening participants, presence of a SSA/P was significantly associated with presence of synchronous adenomas (OR 2.67, $P=0.002$ ). Prevalence of SSA/P was $13 \%$ in FITpositive screening participants, compared with $6 \%$ in control patients. This difference was significant (adjusted OR 1.9, $P=$ 0.01 ) after controlling for age, sex, bowel preparation, colonoscopist and year, but not when controlling for presence of an adenoma (adjusted OR 1.43, $P=0.157$ ) [15]. These data suggest that the higher prevalence of SSA/P in FIT-positive patients is because of a higher prevalence of conventional adenomas in this screening cohort rather than the detection of SSA/P by a positive FIT. This adds weight to the notion that higher minimum expected rates for SSADR and PSLDR would be reasonable in countries such as Australia, the UK and some European countries, where patients generally proceed to screening colonoscopy only where there is a significant family history or a positive FIT.

\section{Conclusions}

In this study of 841 colonoscopies over 4 years by a single colonoscopist, with histology reported by a single expert pathologist, the PSLDR of $29.4 \%$ in the final year of the study exceeds previously reported detection rates. SSADR and PSLDR improved each year with experience, suggesting a learning effect. The current recommendation regarding the minimum PSLDR of $4.5 \%$ in average-risk screening populations should be reevaluated in different patient populations. Consideration should be given to increasing the minimum PSLDR in countries where screening colonoscopies are carried out on patients with increased CRC risk (family history or positive FIT), and not on the average-risk population. Consideration should also be given to exploring PSLDR as a new quality marker in screening and surveillance colonoscopy. Further studies are required to establish 
whether targeted educational interventions can improve PSLDR and whether a high PSLDR does in fact correlate with decreased rates of interval CRC, or whether it remains of academic interest only.

Competing interests

None

References

[1] Vogelstein B, Fearon ER, Hamilton SR et al. Genetic alterations during colorectal-tumor development. N Engl J Med 1988; 319: 525-532

[2] Kedrin D, Gala MK. Genetics of the serrated pathway to colorectal cancer. Clin Transl Gastroenterol 2015; 6: e84

[3] Rex DK, Ahnen DJ, Baron JA et al. Serrated lesions of the colorectum: review and recommendations from an expert panel. Am J Gastroenterol 2012; 107: 1315-1329 quiz 4, 30

[4] East JE, Vieth M, Rex DK. Serrated lesions in colorectal cancer screening: detection, resection, pathology and surveillance. Gut 2015; 64: $991-1000$

[5] Australian Cancer Network Colorectal Cancer Guidelines Revision Committee. Guidelines for the Prevention, Early Detection and Management of Colorectal Cancer. Sydney: The Cancer Council Australia and Australian Cancer Network; 2005

[6] Cancer Council Australia Colonoscopy Surveillance Working Party. Clinical Practice Guidelines for Surveillance Colonoscopy - in adenoma follow-up; following curative resection of colorectal cancer; and for cancer surveillance in inflammatory bowel disease. Sydney: Cancer Council Australia; 2011

[7] Rex DK, Boland CR, Dominitz JA et al. Colorectal Cancer Screening: Recommendations for Physicians and Patients From the U.S. MultiSociety Task Force on Colorectal Cancer. Gastroenterology 2017; 153 : $307-323$

[8] le Clercq CM, Mooi RJ, Winkens B et al. Temporal trends and variability of colonoscopy performance in a gastroenterology practice. Endoscopy 2016; 48: $248-255$

[9] Bettington M, Walker N, Rahman T et al. High prevalence of sessile serrated adenomas in contemporary outpatient colonoscopy practice. Intern Med J 2017; 47: 318-323

[10] Kahi C], Hewett DG, Norton DL et al. Prevalence and variable detection of proximal colon serrated polyps during screening colonoscopy. Clin Gastroenterol Hepatol 2011; 9: 42-46

[11] Dwyer J, Churchill T, Manthe $M$ et al. Factors influencing sessile serrated adenoma (SSA) detection rates at a multi-centre metropolitan Australian health service. J Gastroenterol Hepatol 2014; 29: 27-45

[12] Kaminski MF, Thomas-Gibson S, Bugajski M et al. Performance measures for lower gastrointestinal endoscopy: a European Society of Gastrointestinal Endoscopy (ESGE) Quality Improvement Initiative. Endoscopy 2017; 49: $378-397$

[13] Rex DK, Ponugoti PL. Calculating the adenoma detection rate in screening colonoscopies only: Is it necessary? Can it be gamed? Endoscopy 2017; 49: 1069-1074

[14] Kaminski MF, Regula J, Kraszewska E et al. Quality indicators for colonoscopy and the risk of interval cancer. N Engl J Med 2010; 362: $1795-1803$

[15] Tutticci NJ, Leggett B, Appleyard MN et al. The prevalence of sessile serrated adenomas in a fecal immunochemical test positive colorectal cancer screening cohort. Gastrointest Endosc 2012; 75: AB342 